\title{
A New Unicast Routing Algorithm for Load Balancing in Multi- Gateway Wireless Mesh Networks
}

\author{
Mohammad Karim Jafaryan', Mohammad Ali Jabraeil Jamali², Shahin Akbarpour ${ }^{3}$ \\ ${ }^{1}$ Faculty of Engineering, Department of Computer ,East Azarbaijan Science and Research Branch, Islamic \\ Azad university, Tabriz, Iran \\ ${ }^{2,3}$ Department of Computer, Shabestar Branch, Islamic Azad University, Shabestar, Iran \\ E-mail: 'mohammad.jafaryan@gmail.com, ${ }^{2}$ m_jamali@csri.ac.ir, ${ }^{3}$ sh_akbarpour@csir.ac.ir
}

\begin{abstract}
Gateways are a very important element of wireless mesh networks. They provide the connection between the whole network and the outside world, including the Internet. Since all of input and output traffic in the network go through gateways, these points are simply prone to congestion. Despite having multiple gateways in the network, in the absence of load balancing in the network, some of these gateways are still in danger of congestion that causes traffic aggregation in gateways, and hence, degrading of network throughput. In this paper we propose a method named LBMGW for selecting appropriate gateways that aims load balancing in gateways and increasing network performance. LBMG is the measure for selecting a gateway which is a combination of measures such as negative percent hop, time, and internal load in each gateway. Furthermore, some conditions have been considered to prevent control overhead inside the network. GloMoSim has been used for simulations. Then the results are compared with other available methods that show improvement in throughput of wireless mesh networks with the proposed method.
\end{abstract}

Keywords: Wireless Mesh Network, Gateway load Balancing, Negative Percent Algorithms.

\section{INTRODUCTION}

Recently, Wireless Mesh Networks [1, 2] (WMN) has received attention as a new generation of wireless networks for providing better access service. Figure 1 illustrates a Wireless Mesh Network including some Mesh Gateway (MG), Mesh Router (MR), and Station (STA) [3] nodes.

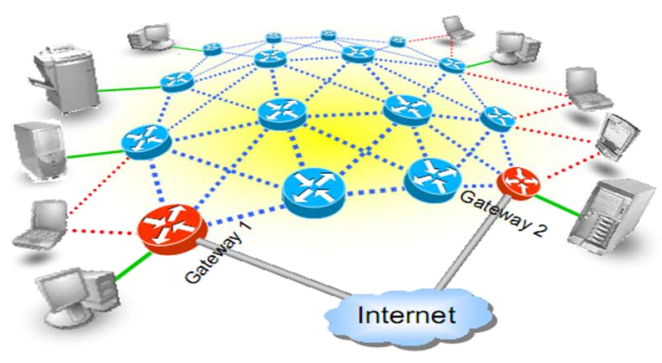

Fig. 1. A Wireless Mesh Network
WMN is an economic solution to provide broadband wireless access to the Internet for residential areas. IEEE $802.11 \mathrm{~s}$ is an IEEE 802.11 [7] amendment for mesh networking, defining how wireless devices can interconnect to create a Wireless Local Area Network (WLAN) mesh network. This architecture of wireless networks emerged to provide Internet access. MR nodes are connected to STA nodes and forward data. STA nodes can be any terminal device such as a computer, cell phone, lap top, or etc. These nodes send the data to MR nodes which, in turn, send the data to gateways through intermediate MR nodes. Finally, gateways send the data to outside world or the Internet. Since all input and output network traffic go through these nodes, they could simply become overloaded and congested. It is possible to distribute the traffic among other gateways by increasing the number of gateways, but in the absence of load balancing in the network, some of these gateways are still congested. Concentration of traffic and lack of load balancing in a specific part 
of the network cause congestion, packet loss, and queue overflow at that part [5]. It is possible to balance network traffic, and also increase network throughput and performance by combining different measures.

\section{RELATED WORKS}

A lot of works have been done about load balancing in wireless networks. The proposed methods are usually different from each other in terms of gateway discovery method (reactive [10]/proactive [11]) and various measures. In Ref [4], a method has been proposed in which in case of excessive packet drops (more than a threshold) a gateway detects the active source and asks the source to send its traffic to other gateway. In Ref [5], another method has been proposed that uses a combination of wired and wireless networks to select the appropriate gateway. MR nodes select the desired gateway based on the orders received from the central dispatcher on the wired side of the network. ETX [9] is the measure to select the route in central dispatcher. In Ref [6], a method called Back Bone Selection (BGS) is proposed that is a combination of Link Interface Factor (LIF), Expected Link Quality (ELQ) [8], and hop count measures. Although the combination of measures leads to better load balancing, it increases routing overhead. In Ref [10], a distributed method is used and the measure for this algorithm is a combination of bandwidth utilization and hop count. In TBMGA protocol (the base of our proposed method), each gateway broadcasts a Root Announcement (RANN) message to the network in constant intervals. This message is received by all mesh nodes. Each node selects the best message (among all of received RANN messages), and sends a RREP message towards the gateway. Therefore, each mesh node builds its own tree route to the gateway and sends its packets through the route.

Each mesh node compares RANN messages based on the ETX measure. The mesh node checks the ETX measure of the RANN message. If the measure is better than previous measures then it will be stored by the node. Otherwise, the node discards the packet. In the end, each mesh node has selected its best gateway which is a new RANN with the best measure. Anyway, to ensure a bidirectional connection, the gateway has to also be aware of new routes to reach mesh nodes. To do so, each mesh node - after selecting its main gateway (based on the ETX measure) - sends a RREP message to the gateway through the new route.
Once the gateway receives the message, it updates its routing table and detects the nodes in its neighborhood.

But, after a while it is possible that some queues of a gateway become congested (compared to other gateways). In that case, the congestion must be distributed among other gateways. To have a balanced load among the control nodes, the average of gateways' queue congestion must not exceed a threshold. In the case of exceeding the threshold, the gateway must find one of the connected mesh nodes with the highest value and send a CHANGEPKT message to it and ask the node to find another gateway (if that is possible) for transmitting its packets. Once the mesh node receives the CHANGE-PKT message, it sends a GW-REQ to other gateways. Those gateways that receive the message and can accept a new MN responds to it with a GW-REP message. Then the MN will be an associate of the first respondent gateway. Once a GW-REP is arrived at a MN, the node sends all of its extra traffic towards its new gateway, but if the traffic is not accepted by any gateway, the MN node continues sending its packets towards its last gateway. The first drawback of this method is that when a gateway sends a CHANGE-PKT to a node and changes the route of that node, it cannot anticipate the status of queue congestion at other gateways compared to its own queue. Thus, each node has to send some extra messages to other gateways. It is possible that the queues of other gateways are much busier than the default gateway, and hence, the packets are sent towards the last gateway which will possibly be rejected by that gateway and causes excessive rush. The second drawback is if a CHANGE-PKT causes a node to change its route, leaf nodes of that node also change their route. In other words, a CHANGEPKT can change multiple nodes at the same time, and other gateways reject the MN. Thus, finding the best node for change and separating from the gateway has to be in such a way that does not cause a problem for other gateways. Changing the gateways might forms a ping-pong state. It means other gateways suffer from congestion and send CHANGE-PKT. If this situation continues the network becomes unstable. Sending CHANGEPKT has to be done periodically not simultaneoussly, because if the congestion at all gateways exceeds the threshold at the same time, the network will be flooded by CHANGE-PKT messages, and hence, all mesh nodes have to send their packets towards their former gateway which is an unstable situation. 


\section{THE PROPOSED METHOD}

In the proposed method, each mesh node which is recognized as a gateway starts the route discovery periodically. Each gateway broadcasts a HelloPacket message to the network. Each node that receives the message sends a RREP message to the gateway. This allows the gateway and the node to have a bidirectional connection without any need to an already established route (proactive). Once a mesh node receives a Hello-Packet message, it creates its route towards the gateway or updates the existent entry (only in the case that sequence number of the Hello-Packet is greater than current route, or sequence numbers are the same, but the node has a better measure than the current route). Since the route measure between the source and the destination is cumulative calculated), each intermediate mesh node has to update the route discovery measure field in the Hello-Packet. Once a mesh node receives Hello-Packet messages, it sends the RREP message to the gateway.

\subsection{The Proposed Algorithm}

Assume that to provide an appropriate capacity in the network a new gateway node is added to the WMN. At first, the new gateway broadcasts a Hello-Packet message and announces its presence. Then, the gateway constantly broadcasts the message in specific intervals. This leads to a proactive gateway discovery at other mesh nodes. Once a mesh node receives the message, it adds a new row to its gateway table and records its specifications. Then, mesh nodes select the best message based on predetermined measures. In traditional methods that are inspired by ad hoc networks, route-relevant measures such as hop count are usually used which is not a suitable measure to select the best gateway. In the proposed method, a combination of route-relevant measures and gateway-relevant measures are used to select the gateway. The mesh router selects its default gateway based on route and gateway traffic information and updates its access route to the Internet. Negative-percent time and negativepercent hop are used as measures for route information. Moreover, negative-percent load (number of available links at the gateway, i.e., number of MR nodes that the gateway is currently serving) is used as the gateway-relevant measure. Once a Hello-Packet is arrived at a MR node, the node updates its list of gateways and selects the gateway with the lowest measure value.

\subsection{Introducing Gateway Selection Measures}

Load measure: Each gateway advertises its current load (number of mesh nodes that are currently served by the gateway) to other nodes. A mesh node calculates a negative percentage for the gateway based on the load, and selects the lowest percentage because the gateway with the lowest negative percentage can receive more traffic. Relation 3-1 shows the load measure.

$$
\begin{aligned}
& \text { negative }- \text { percentloadmeasureof } \boldsymbol{i} \\
& \text { gatewayfor } \boldsymbol{M} n=L w_{\text {im }}{ }^{1} \text { loadofigateway }=l_{i} \\
& 1<i<n, \quad n=\text { numberofgateways } \\
& L w_{i m}=\frac{l_{i}}{l_{1}+l_{2}+\cdots+l_{\mathrm{n}}} \quad(3-1)
\end{aligned}
$$

Hop measure: The percentage of load is not merely enough to select the best gateway, because if the distance from a mesh node to the $\mathrm{N}$ gateway is one hop, and to the M gateway is multiple hops, we have to also consider this distance (hop count) to select the gateway. So we calculate hop counts from current mesh node to the gateway. A negative percentage of hop counts is derived which is used as the second measure. Relation 3-2 shows the hop measure.

$$
\begin{gathered}
\text { negativepercenthopmeasureof } \\
\quad \text { igatewayforMnode }=h w_{i m}{ }^{2} \\
\text { hopcountofigateway }=h_{i} \\
1<i<n, \quad n=\text { numberofgateways } \\
h w_{i m}=\frac{h_{i}}{h_{1}+h_{2}+\cdots+h_{n}} \quad(3-2)
\end{gathered}
$$

Time measure: Each data packet that is sent by the gateway spends some time to arrive at a mesh node. So if the distance between two gateways is equal then we should also enter the time into our calculations. By calculating a negative percentage of these times, we assign a third measure (time) to each gateway. Relation 3-4 shows the negative percent time measure.

$$
\begin{gathered}
\text { negative - percenttimemeasureof } \\
\text { igatewayforMnode }=t w_{i m}{ }^{3} \\
\text { calculatedtimetoigatewa }=t_{i}
\end{gathered}
$$

\footnotetext{
${ }^{1}$ Load Weight of I gateway for M node

${ }^{2}$ Hop Weight of I gateway for M node

${ }^{3}$ Time Weight of I gateway for M node
} 
M. K. Jafaryan et al. / International Journal of Computer Networks and Communications Security, 1 (6), November 2013

$$
\begin{gathered}
1<i<n, \quad n=\text { numberofgateways } \\
\mathrm{tw}_{\mathrm{im}}=\frac{\mathrm{t}_{\mathrm{i}}}{\mathrm{t}_{1}+\mathrm{t}_{2}+\cdots+\mathrm{t}_{\mathrm{n}}} \quad(3-3)
\end{gathered}
$$

Finally, we have the new measure $\mathrm{LBMGW}^{4}$ by combining all previous measures which is shown in the following relation. MR nodes select the gateway having the lowest LBMGW.

\section{(3-4) - the new measure for the proposed protocol}

$$
\mathrm{W}_{\mathrm{LBMGW} 1_{\mathrm{mi}}}=\mathrm{tW}_{\mathrm{mi}}+\mathrm{hW}_{\mathrm{mi}}+\mathrm{lW}_{\mathrm{mi}}
$$

(3-5) - calculating the best gateway without considering the weight

$G W_{M}=\min \left\{W_{L B M G W_{1} M_{1}}, W_{L B M G W_{1} M_{2}}, \ldots, W_{L B M G W_{1} M_{n}}\right\}$

With a low congestion, load has a more effective impact because duration of send and receive is varying. At high congestion that more time is needed, we represent the load with a coefficient to keep the effect of the load. This helps a mesh node to stay away from a gateway with more congestion. The more the number of users (links) is, the more effective this staying away will be. Since the Internet users use gateways, we are trying to direct the new user towards the gateway with less number of users by increasing number of users to help the network not lose its efficiency at high congestion.

(3-6) - calculating the new measure for the proposed protocol considering time weight

$\mathrm{W}_{\mathrm{LBMGW} 2 \mathrm{mi}}=\mathrm{tW}_{\mathrm{mi}}+\mathrm{hW}_{\mathrm{mi}}+\mathrm{lW}_{\mathrm{mi}} \times \mathrm{tW}_{\mathrm{mi}}$

(3-7) - calculating the best gateway without considering time weight

$G W_{M}=\min \left\{W_{L B M G W_{2} M_{1}}, W_{L B M G W_{2} M_{2}}, \ldots, W_{L B M G W_{2} M_{n}}\right\}$

Each Hello-Packet is broadcasted at a specific time that causes an extra overhead on all network routes. The following method is used to decrease the overhead of sending Hello-Packet messages. Upon receiving a Hello-Packet, each router first compares the packet with the last table. If new packet information is better than the old one, the entry is updated and the router broadcasts the packet. Other intermediate nodes do the same. On the other hand, if the current Hello-Packet is not better than the last one, the router updates the entry but does not broadcast the packet. This causes some mesh nodes do not broadcast Hello-Packets. So

\footnotetext{
${ }^{4}$ Load Balancing Multi Gateway Wireless meshnetwork
}

intermediate nodes do not receive these packets, and hence, the overhead is decreased.

\section{SIMULATION AND RESULTS}

In this section, performance of the proposed LBMGW method is evaluated against the TBMGA method in terms of average data transferred, packet delivery ratio, control overhead, and average power consumption. Simulations are carried out by GloMoSim network simulator which provides a suitable environment for simulating wireless communications.

\subsection{Simulation Parameters}

We assume that all links have the same capacity. Transmission range for all nodes is 250 meters. Each simulation lasts 300 seconds. Number of 2060 mesh nodes and 2-8 gateways are used. Simulation area is $1000 \times 1000$ meters and IEEE 802.11 is used as the MAC protocol. FTP and TELNET are chosen as traffic patterns. IP is the network protocol.

\subsection{Quality of Service Comparison (LBMGW VS. TBMGA)}

Figure 2 shows the average delay for both protocols. With a fixed and variable number of nodes the proposed method has the lowest delay in sending data packets.
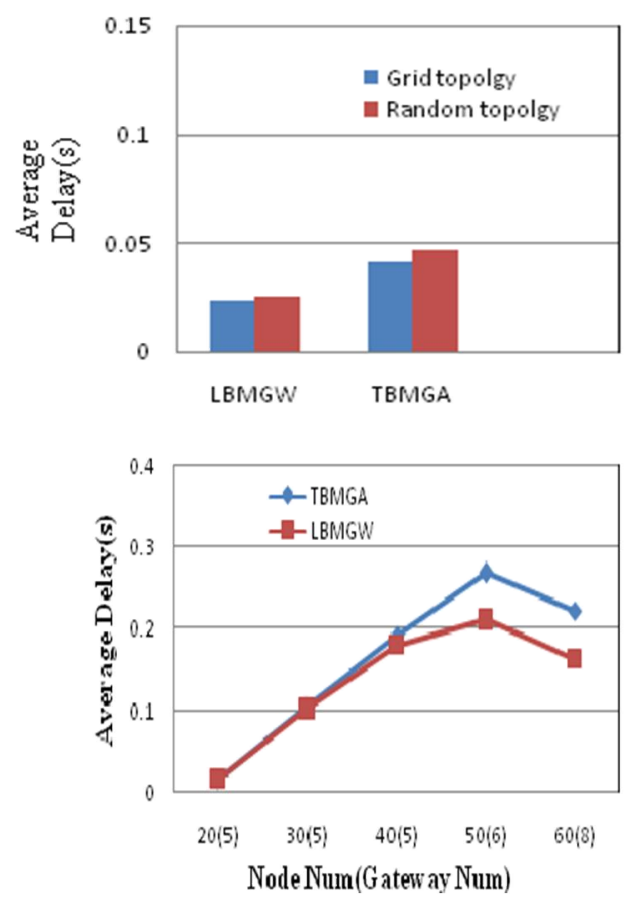

Fig. 2. Delay 
In the proposed method, each intermediate node imposes a short delay on sending packets. It is probable that some packets will not be delivered since, possibly, the link between one of the intermediate nodes and other nodes is broken for some reasons. Figure 3 verifies this possibility.

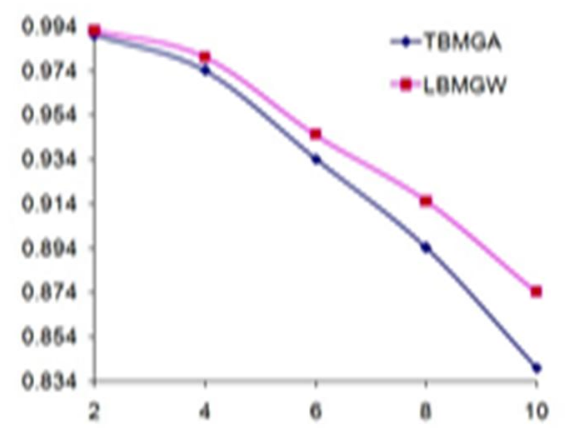

Fig. 3. Packet delivery ratio with a variable number of senders

In Figure 4, packet delivery ratios of both protocols with a variable number of gateways are compared. As it shows, packet delivery ration rises while number of gateways increase from 2 to 3 .

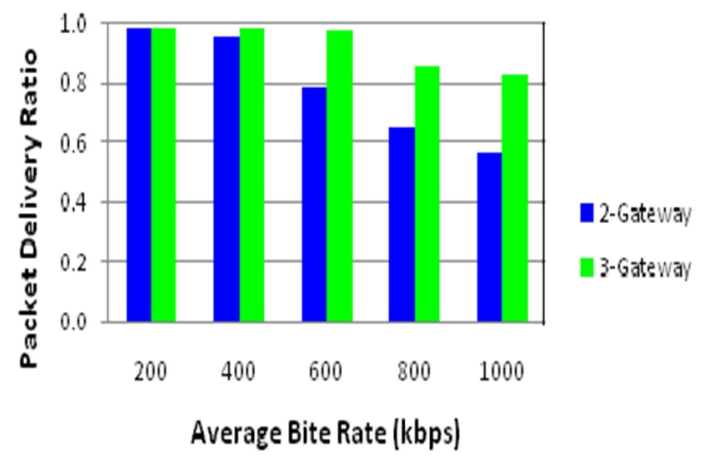

Fig. 4. Packet delivery ratio with a variable number of gateways

Figure 5 shows that in the case of fixed and variable number of nodes, the proposed method has a less control overhead than the TBMGA method. This is because the proposed method avoids sending control packets at some intervals. So mesh nodes do not receive these packets. This situation is investigated in both mesh and random topology.
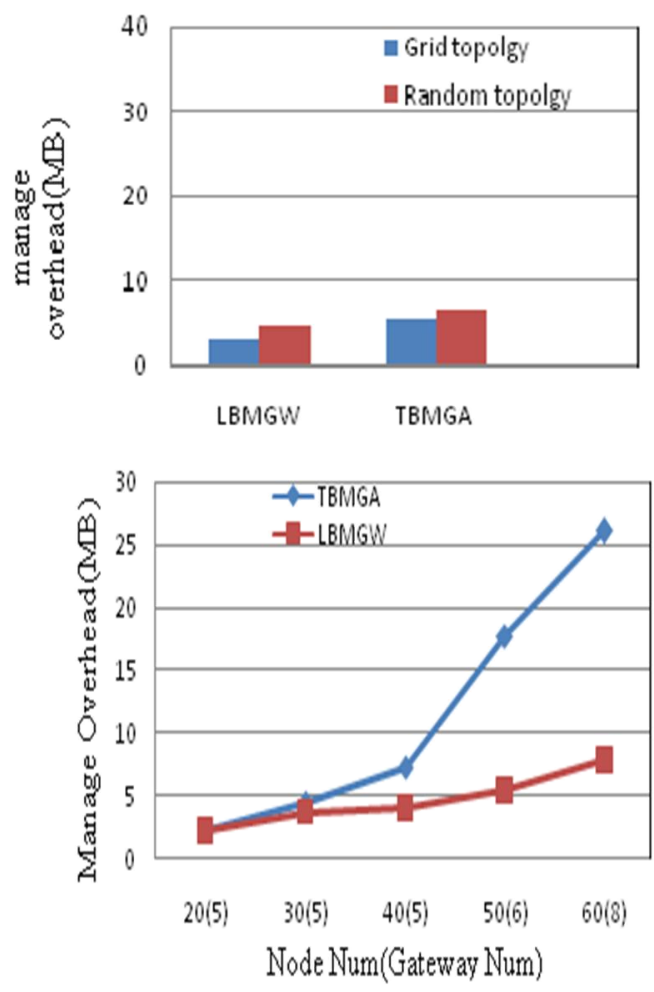

Fig. 5. Control overhead

The effects of network growth and traffic flows on average power consumption in the case of stationary nodes are shown in Figure 6. By using the proposed method, number of internetwork transmissions is decreased (compared to other methods). Thus, wireless nodes keep their power longer, and hence, their energy is saved.

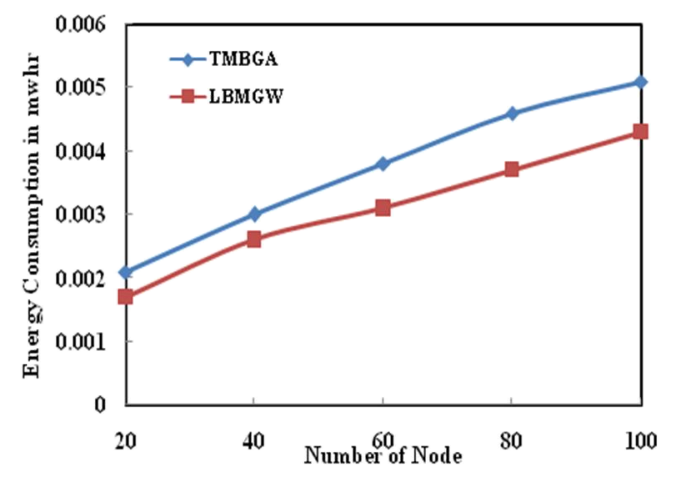

Fig. 6. Average power consumption with number of nodes 


\section{CONCLUSION}

In this paper we have proposed an algorithm for load balancing. The proposed method is seeking for a load-balancing-based solution to decrease overhead and delay, and increase network throughput. Simulation results show that with the LBMGW proposed method, if we take both route and gateway status into account, we can present a measure to perform load balancing well by uniformly directing traffic flows through the network and available gateways. It improves network capacity and decreases end-to-end delay compared to other methods.

\section{REFERENCES}

[1] Akyildiz, I. F., Wang, X., and Wang,W., "Wireless Mesh Networks: Survey", Computer Networks, Vol. 47, No. 4, pp. 455-487, March 2005.

[2] Whitehead, P., "Mesh Networks: A New Architecture for Broadband Wireless Access Systems", Proceedings of IEEE Conference on Radio and Wireless (RA WCON), pp. 43-46, 2000.

[3] IEEE P802.11 Task Groups, IEEE Unapproved draft standard P802.11s/D7.0, July 2010.

[4] Nandiraju, T., Efficient Traffic Diversion and Load-balancing in Multi-HopWireless Mesh Networks", Master of Science (Computer Science)Assam University,Silchar, India, 2003.

[5] LIN, P., YEUNG, K. H., WONG, K., Y., Multiple Path Routing using Tree-Based Multiple Portal Association for Wireless Mesh Networks

[6] Ashraf, U., Abdellatif, S., Juanole, G., Gateway Selection in Backbone Wireless Mesh Networks, Proceeding of Wireless Communications and Networking Conference, 2009.

[7] Xiea, B., Yub, Y., Kumarc, A., Agrawala, D. p., "Load-balanced mesh router migration for wireless mesh networks", Journal of Parallel and Distributed Computing vol. 68, Issue 6, pp. 825-839, 2008.

[8] Ashraf, U., Abdellatif, S., Juanole, G., An Interference and Link-Quality Aware Routing Metric for Wireless Mesh Networks, in 68th IEEE Vehicular Technology Conference (IEEE VTC 2008) Sept 2008.

[9] Couto, D.S.J., Aguayo, D., Bicket, J., Morris, R., "A High Throughput Path Metric for Multi-Hop Wireless Routing",
Wireless Networks, vol.1l, iss.4, pp.419-434, Jul.2005

[10] Garroppoa., G.R., Giordanoa, S.,Tavanti, L., "Implementation frameworks for IEEE 802.11 s systems", Computer Communications, vol. 33, no. 3, pp. 336-349, 2010.

[11]Bahr, M., Update on the Hybrid Wireless Mesh Protocol of IEEE802.11s, in 1st IEEE International Workshop on Enabling Technologies and Standards for Wireless Mesh Networking (MeshTech 2007), Pisa (Italy), Oct. 2007. 